\title{
TRANSVERSALIDAD DE PERSPECTIVA DE GÉNERO EN LA UNIVERSIDAD DE SONORA: NORMATIVIDAD Y PERCEPCIÓN DE LOS ALUMNOS SOBRE PROCESOS DE SENSIBILIZACIÓN E INSTITUCIONALIZACIÓN. UNA PROPUESTA DE INNOVACIÓN EDUCATIVA.
}

Jesús Miguel MAYA RODRÍGUEZ ${ }^{1}$

Emilia CASTILLO OCHOA ${ }^{2}$

SUMARIO: I. Introducción. II. Planteamiento del Problema. III. Justificación. IV. Objetivo General. V. Dimensión, Variables e Indicadores propuestos. VI. Esbozo General del Método. VII. Posibles aportaciones. VIII. Referencias Bibliográficas.

Este artículo plantea la necesidad y obligación para las Instituciones de Educación Superior en México de realizar investigación en la línea de educación y género, en el sentido de la transversalidad de la perspectiva de género, ante una sociedad en la que cada vez pesa más un sentido de exclusión e indiferencia hacia las desigualdades con los grupos vulnerables. Se pretende exponer las bases de una investigación que se realizara en la Universidad de Sonora, en México, en el marco de estudios en el programa de Maestría en Innovación Educativa, la cual considera tener un alcance descriptivo, a través del análisis del problema desde las variables de normatividad, sensibilización, institucionalización y demográficas, que permita estar en posibilidad de elaborar una propuesta de innovación educativa, y en consecuencia formar profesionales no solo científicos, sino "más" humanos y comprometidos con la justicia que constituye el reclamo social.

\footnotetext{
${ }^{1}$ Licenciado en Derecho por la Universidad de Sonora, Maestrante en Innovación Educativa, Universidad de Sonora.

${ }^{2}$ Profesora de Tiempo Completo Titular, Departamento de Psicología y Ciencias de la Comunicación, Universidad de Sonora. 
Palabras Clave: Equidad de Género, Transversalización, Educación Superior, Perspectiva de Género.

\section{Introducción}

Este articulo, pretende exponer los datos y justificación que da origen a una propuesta de investigación a realizarse en la División de Ciencias Sociales de la Universidad de Sonora, en la Unidad Regional Centro, analizando la transversalización de la perspectiva de género en los estudiantes de programas de licenciatura, desde variables especificas que se proponen. Esta idea se plantea ante la inexistencia de estudios de género realizados en la institución, que ofrezcan una radiografía aunque limitada, en cierto modo certera y de carácter exploratorio, que nos lleve a observar el fenómeno de la equidad de género, y con la información recabada generar propuestas que impliquen a algunos aspectos de las actividades sustantivas de la Universidad de Sonora, colaborando a la formación integral del estudiante, y las actividades de la institución, dentro del esquema de la educación superior y las respuestas que demanda la sociedad de los egresados de la misma

\section{Planteamiento del Problema}

Para lograr entender el objeto del problema a investigar se hace necesario partir definiendo que a la transversalización de la perspectiva de género podemos entenderla como "'Incorporar el enfoque de equidad de género de forma transversal en todas las políticas, estrategias, programas, actividades administrativas y económicas e incluso en la cultura institucional de una organización para contribuir verdaderamente a un cambio en la situación de desigualdad genérica". (ONU, 2004, p. 6), por lo que llevada la anterior conceptualización al campo de las Instituciones de Educación Superior, consistiría 
en su observancia e integración a los planes institucionales de desarrollo, los marcos normativos al interior de las instituciones bajo el goce de la autonomía universitaria, a los procesos de gestión administrativa, la aplicación de los recursos económicos, los planes y programas de estudio, así como los de formación continua, actividades formativas extracurriculares, como pueden ser las prácticas profesionales, el servicio social obligatorio, la praxis docente, el currículo formal y las implicaciones referentes al currículo oculto, los procesos de formación integral, las actividades de investigación, desarrollo, y la difusión de los conocimientos generados al interior de sus muros.

En el contexto de lo anterior el Estado Mexicano, se compromete en el ámbito de la comunidad internacional con la firma de compromisos con organismos como la Organización de las Naciones Unidas (2004, p. 3), en el cual plantea a través de los objetivos del desarrollo del milenio numeral 3, el "promover la igualdad de género y autonomía de la mujer", como ideal que deberán consideran los estados firmantes. Otra es la Organización de las Naciones Unidas para la Educación, la Ciencia, y la Cultura (2008) en la que el Priority Gender Equality Action Plan 2008-2013, se determinan directrices en los campos de la educación, la ciencia, y la cultura para la promoción de la equidad de la mujer. Por otra parte en la Organización de las Naciones Unidas para la Igualdad de Género y el Empoderamiento de la Mujer en la Convención para la Eliminación de todas las Formas de Discriminación contra la Mujer (1979), describe parámetros que impulsen la erradicación de la discriminación por género y fortalezcan el empoderamiento de la mujer con acciones específicas de los gobiernos.

A su vez estos compromisos se plasman en las Políticas Públicas y el marco normativo regulatorio. El Gobierno de los Estados Unidos Mexicanos, la Presidencia de la Republica (2007, p. 85) en el Plan Nacional de Desarrollo 20072012 en su eje 3 de igualdad de oportunidades, prioriza y enfoca las acciones y esfuerzos del estado a la reducción de la brecha desigualitaria, hacia una equidad de género en la educación, el acceso a la salud y otros aspectos de la vida 
pública, retomándose esta política en el Programa Sectorial de Educación 20072012 de la Secretaría de Educación Pública (2007, p. 11).

Ante estas pautas, en el año 2006 el Poder Legislativo -Órgano de poder encargado de la creación de las leyes en México-, creo la Ley General para la Igualdad entre Mujeres y Hombres (2011), ordenándose en ello la transversalidad de género en todos los aspectos de la actividad de las instituciones y organismos de la Administración Pública Federal, incluyendo en esto el Sistema Educativo Nacional. Además se crea el Sistema Nacional para la Igualdad entre Mujeres y Hombres, y se dota de mayores facultades y responsabilidades al Instituto Nacional de la Mujer determinando de manera explícita una regulación normativa en materia de igualdad de género para la nación.

Con la finalidad de otorgar solidez a lo propuesto en la precitada norma jurídica, y teniendo en consideración que el presupuesto de gasto público es un elemento idóneo y necesario para impulsar la equidad de género se consideró en el Presupuesto de Egresos de la Federación 2008, que a través del gasto etiquetado consistente este en "recursos que los gobiernos destinan a programas y acciones para atender las necesidades, intereses y derechos de las mujeres y/o promover la equidad de género (Centro de Estudios para el adelanto de las Mujeres y la Equidad de Género, p. 9 ), se consideraron rubros de inversión específicos a la educación en el diseño y aplicación en la política educativa de la transversalización de la perspectiva de género, entre otras acciones (Comisión de Equidad de Género, 2007, p. 6). Este ramo de gasto se ha venido fortaleciendo con las ampliaciones y etiquetaciones propuestas por la Comisión de Equidad y Género de la Cámara de Diputados del H. Congreso de la Unión en el Presupuesto de Egresos de la Federación 2009, proporcionando recursos económicos a las Instituciones Públicas de Educación Superior para la elaboración de programas con perspectiva de género (Comisión de Equidad de Género, 2008).

Al respecto la Asociación Nacional de Universidades e Instituciones de Educación Superior (2000, p. 249), en la propuesta a la educación superior, La Educación superior en el Siglo XXI Líneas estratégicas de desarrollo, reconoce el 
crecimiento exponencial que enfrentara la matrícula de género femenino en las Instituciones de Educación Superior, con lo que se puede considerar que se ha impulsado la transversalización en el aspecto demográfico de matriculación.

En el Estado de Sonora, el Poder Ejecutivo Estatal, a través del Plan Estatal de Desarrollo 2009-2015 (2009, p. 2), adopta igualmente como una Política Pública en el Estado la equidad de género en la Administración Pública. Por su parte la Universidad de Sonora, como Institución Pública y Autónoma de Educación Superior, con personalidad jurídica y patrimonio propios, en su Plan de Desarrollo Institucional 2009-2013 (2009, pp. 76-77), establece como objetivo "Ofrecer igualdad de oportunidades a los estudiantes en el acceso a una formación de calidad y realizar acciones que limiten las situaciones de exclusión social." Definiendo su interés por ser un espacio que ofrezca un trato igual a los sujetos que la integran además de contribuir al desarrollo armónico, y reducción de las diferencias sociales.

Recapitulando lo anterior, se tiene que en vista de los presupuestos asignados por la federación a las Instituciones Públicas de Educación Superior para integrar la transversalidad del género del 2008 a la fecha, y la adopción misma de estos objetivos sectoriales por la Universidad de Sonora en sus políticas internas, por lo que la institución ha implementado algunos procesos de investigación y acciones específicas sobre la temática siendo uno de ellos el proyecto "Estudio de igualdad de género en la Universidad de Sonora a partir de la percepción de los actores de las carreras de la División de Ciencias Sociales" del cual se deriva el presente recorte de investigación cuyo objetivo es "Identificar las percepciones que sobre equidad de género tienen los alumnos, profesores y personal no docente de licenciatura de las carreras adscritas a la División de Ciencias Sociales", de este se deriva el presente, consistente en "Diagnosticar y evaluar, la transversalidad de la perspectiva de género en la Universidad de Sonora como política pública dirigida a los alumnos, en la normatividad y la percepción de estos en los procesos de sensibilización e institucionalización, y a 
partir de las necesidades detectadas diseñar propuesta de innovación para la mejora de las prácticas de igualdad entre hombres y mujeres", ante la situación actual de la Universidad de Sonora donde estas acciones de integración de la transversalidad de la perspectiva de género, que se han llevado a cabo en los procesos sustantivos se desconoce el impacto y consecuencias que han generado a la dinámica de la vida estudiantil y universitaria en general.

\section{Justificación}

La importancia y necesidad de llevar a cabo este tipo de estudios tiene sus fundamentos en los siguientes argumentos:

- Por qué "los estudios de género ha demostrado que la desigualdad entre hombres y mujeres es producto de una construcción cultural y no resultado de capacidades naturales diferenciadas entre los sexos" (Buquet Corleto, 2011, p. 212), lo que implica que los espacios formativos de las Universidades son propicios para producir cambios sustanciales en las prácticas culturales de los sujetos que lo integran, ya que no solo sus esfuerzos se avocan a la formación en aspectos científicos y profesionalizantes que demanda el mercado laboral si no que tiene una "función socializadora y un efecto multiplicador" (Buquet Corleto, 2011, p. 217), con lo que estos estudiantes desempeñan sus actividades en la sociedad de la que forman parte, hoy en día como estudiantes y en el futuro como profesionistas y en algunos casos padres de familia que conformaran la parte estratégica del conglomerado social en formación que será capaz de transformar la desigualdad si se encuentran debidamente preparados para ello o por el contrario reproducirlas y generar otras más.

- Por qué la Universidad de Sonora en su Plan de Desarrollo Institucional 2009-2013 (2009, pp. 76-77) establece "Ofrecer igualdad de oportunidades a 
los estudiantes en el acceso a una formación de calidad. y realizar acciones que limiten las situaciones de exclusión social.", con lo cual la institución se compromete con la incorporación de la Transversalidad de Género en todas sus actividades y acciones de promoción.

- $\quad$ Por qué en la Universidad de Sonora se han realizado pequeños estudios de transversalidad de género, como el elaborado por Rodríguez, Urquidi. y Pérez, (2010), pero aún falta muchos más, entre ellos uno que considere las dimensiones del que se plantea llevar a cabo, lo que hace necesario realizar estas investigaciones ya que "permiten hacer visible la situación en la que mujeres y hombres desarrollan sus actividades académicas y profesionales....detectar los núcleos problemáticos...las necesidades particulares de cada institución". (Buquet Corleto, 2011, p. 216), de ahí la importancia de realizar estudios sobre dimensiones y variables en cada caso y contexto especifico de las Instituciones de Educación que conforman el Sistema de Educación Superior en México.

- Por la obligación de las Instituciones de Educación Superior, de orientar su actuación en el marco de la convivencia de un estado de derecho, basado en normas de observancia obligatoria, originadas de los ideales y aspiraciones de la sociedad, que todas las Instituciones Públicas, incluidas las educativas deben de perseguir como finalidad.

- Se beneficia con este tipo de estudio, el diseño de política pública a partir de los resultados obtenidos y los procesos de inclusión de la perspectiva de género de manera transversal en las Instituciones de Educación Superior, favoreciendo la convivencia social de y entre los actores: alumnas y alumnos en este caso.

\section{Objetivo General}

- Diagnosticar y evaluar, la transversalidad de la perspectiva de género en la Universidad de Sonora como política pública dirigida a los alumnos, en la 
normatividad y en la percepción de estos en los procesos de sensibilización e institucionalización, y a partir de las necesidades detectadas diseñar propuesta de innovación para la mejora de las prácticas de igualdad entre hombres y mujeres.

\section{Objetivos Específicos}

- Realizar breve contextualización de los procesos de transformaciones de la normatividad, desarrollo de la institucionalización y experiencias de sensibilización de la perspectiva de género en las Instituciones de Educación Superior en México.

- Conocer y evaluar en la Universidad de Sonora, el diseño e implementación de políticas para la aplicación de la transversalidad de género en la normatividad, procesos de institucionalización y acciones de sensibilización dirigidas a los alumnos.

- A partir de necesidades detectadas diseñar una propuesta de innovación educativa que coadyuve en los procesos de transversalidad de género, equidad e igualdad de hombres y mujeres en la Universidad de Sonora.

\section{Dimensión, Variables e Indicadores propuestos}

La realización del proyecto, parte de elementos conceptuales clave como son los descritos en la Tabla 1.

Tabla 1. Conceptualizaciones básicas.

\begin{tabular}{|c|c|}
\hline$\frac{\circ}{0}$ & $\begin{array}{l}\text { "A concept which refers to the comparative or differential roles, } \\
\text { responsibilities, and opportunities (all social constructed) of women and } \\
\text { men in a given society". (Dawson, 2006, p. 23). }\end{array}$ \\
\hline
\end{tabular}




\begin{tabular}{|c|c|}
\hline 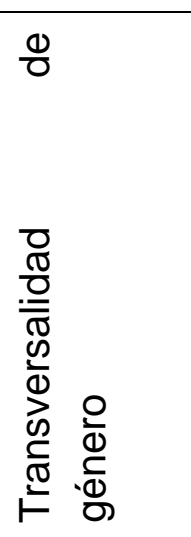 & $\begin{array}{l}\text { "Incorporar el enfoque de equidad de género de forma transversal en } \\
\text { todas las políticas, estrategias, programas, actividades administrativas } \\
\text { y económicas e incluso en la cultura institucional de una organización } \\
\text { para contribuir verdaderamente a un cambio en la situación de } \\
\text { desigualdad genérica". (ONU, 2004, p. 6). Otra definición es la de } \\
\text { Dawson (2006, p. 23) considerándolo como "A process of ensuring that } \\
\text { all policy, programme, administrative, and financial activities contribute } \\
\text { to gender equality, by transforming the balance of power between } \\
\text { women and men". }\end{array}$ \\
\hline 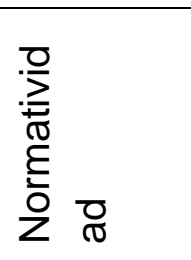 & $\begin{array}{l}\text { Se presenta en el espacio jurídico en dos sentidos, amplio y estricto: } \\
\text { "lato sensu aplicase a toda regla de comportamiento, obligatoria o no, } \\
\text { stricto sensu corresponde a la que impone deberes o confiere } \\
\text { derechos" (García, 2005, p. 4). }\end{array}$ \\
\hline 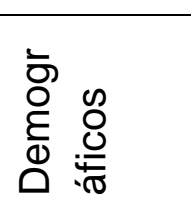 & $\begin{array}{l}\text { En el sentido formal consiste en: "the statistical description and } \\
\text { analysis of human populations; it aims at statistical measurement of a } \\
\text { given population and its evolution" (Overbeek, 1982, p. 2) }\end{array}$ \\
\hline 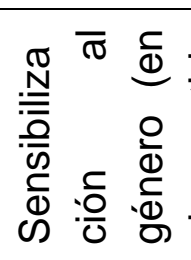 & $\begin{array}{l}\text { "Policies, programs, and plans do in fact identify gender relations as } \\
\text { problematic and offer opportunities for an examination and discussion } \\
\text { of gender issues. They deal with strategic as well as practical" (Leach, } \\
2003, \text { p. 22). }\end{array}$ \\
\hline 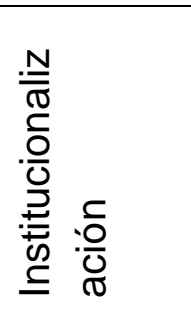 & $\begin{array}{l}\text { "integración sistemática de la igualdad de género en todos los sistemas } \\
\text { y estructuras, políticas, programas, procesos y proyectos para } \\
\text { convertirla en maneras de ver y hacer, en la cultura y sus } \\
\text { organizaciones" (Rees, } 2002 \text { citado por Maceira, Alva y Rayas, 2007, } \\
\text { p. 186) }\end{array}$ \\
\hline
\end{tabular}

Considerando como dimensión global del estudio la tranversalización de la perspectiva de Género en la Universidad de Sonora, se plantea el estudio desde las variables de Normatividad, Demográficas, Institucionalización, y sensibilización, en las cuales se pretende recolectar la información propuesta en los indicadores (Ver Tabla 2).

Tabla 2 Dimensiones, Variables e Indicadores. 


\begin{tabular}{|c|c|c|}
\hline Dimensión & Variables & Indicadores \\
\hline \multirow{4}{*}{ 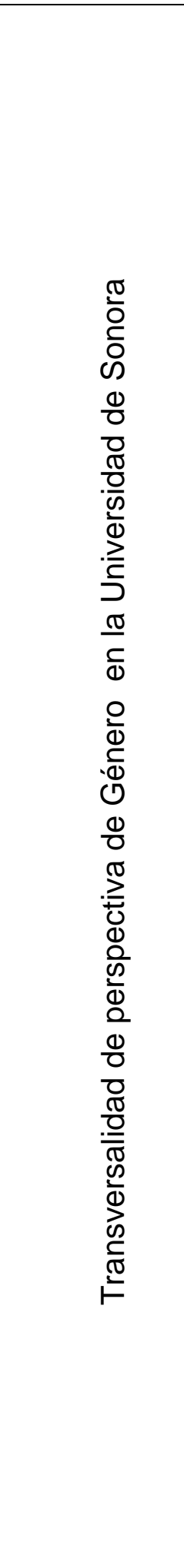 } & Normatividad & $\begin{array}{l}\text { - Normas jurídicas federales y estatales, } \\
\text { vinculadas al género y la educación. } \\
\text { - Planes y programas sectoriales } \\
\text { específicos a nivel federal y estatal. } \\
\text { - Planes y programas sectoriales } \\
\text { específicos en la Universidad de Sonora. } \\
\text { - Presupuestos de egresos e ingresos de } \\
\text { la Federación de apoyo a la } \\
\text { implementación de políticas de género y } \\
\text { educación. } \\
\text { - Presupuestos de egresos e ingresos del } \\
\text { Estado de apoyo a la implementación de } \\
\text { políticas de género y educación. } \\
\text { - Presupuestos de egresos e ingresos de } \\
\text { la Universidad de Sonora de apoyo a la } \\
\text { implementación de políticas de género y } \\
\text { educación. }\end{array}$ \\
\hline & Demográficos & $\begin{array}{l}\text { - Sexo. } \\
\text { - } \text { Estado Civil. } \\
\text { - } \text { Matricula. } \\
\text { - Distribución de alumnas y alumnos por } \\
\text { carrera. } \\
\text { - Aprobación y reprobación de alumnas y } \\
\quad \text { alumnos. }\end{array}$ \\
\hline & Institucionalización & $\begin{array}{l}\text { - Desarrollo de los estudios de género y de } \\
\text { - } \text { la mujer. } \\
\text { inveación de áreas dedicadión de estudios de género. } \\
\text { - Grupos de investigación. } \\
\text { - Organizaciones de estudios de género } \\
\text { - Diseño e implementación de programas } \\
\text { dirigidos a alumnos de perspectiva de } \\
\text { género. } \\
\text { - Procesos de vinculación y colaboración } \\
\text { para implementar acciones de } \\
\text { perspectiva de género para alumnos. }\end{array}$ \\
\hline & $\begin{array}{l}\text { Sensibilización al } \\
\text { género (en el } \\
\text { sentido de } \\
\text { adopción) }\end{array}$ & $\begin{array}{l}\text { - } \text { Conocimiento del tema. } \\
\text { - Medios y estrategias. } \\
\text { - Participación. } \\
\text { - Evaluación. } \\
\text { - Necesidades. }\end{array}$ \\
\hline
\end{tabular}




\section{Esbozo General del Método}

Se pretende desarrollar la investigación bajo el diseño metodológico propuesto en la Tabla 3.

Tabla 3 Concentrado de diseño metodológico

\begin{tabular}{|c|c|}
\hline Alcance de la Investigación & Descriptiva \\
\hline \multirow{2}{*}{ Diseño de investigación } & Cuantitativo \\
\cline { 2 - 2 } & No Experimental - Transeccional \\
\hline Técnica instrumental & Encuesta \\
\hline Límite espacial & $\begin{array}{c}\text { Universidad de Sonora - Unidad Regional Centro- } \\
\text { División de Ciencias Sociales }\end{array}$ \\
\hline $\begin{array}{c}\text { Límite Temporal (aplicación } \\
\text { de instrumento) }\end{array}$ & Abril - Mayo 2012 \\
\hline Población & Estudiantes de Programas de Licenciatura \\
\hline
\end{tabular}

\section{Posibles aportaciones}

Entre algunos de los beneficios que se esperan obtener con este estudio se encuentran :

- Permitirá conocer los avances, fortalezas y necesidades en el proceso de transversalización de la equidad de género en la Universidad de Sonora a partir del marco normativo y la percepción de los alumnos sobre los procesos de institucionalización y sensibilización.

- Posibilitara emitir propuesta de innovación respecto a las variables, que potencialice e impulse el proceso de transversalización en la Universidad de Sonora. 
- Generara información precisa de los alumnos respecto a su "visión" de la igualdad y equidad de género, que viven y desarrollan en los espacios de la universidad.

- Permitirá ampliar el conocimiento y comprensión del fenómeno discriminatorio del género, en la percepción de los alumnos.

- Colaborara al direccionamiento de políticas, y normatividades encaminadas al fortalecimiento del tejido social dentro y fuera de la institución.

- Fortalecer la función formativa, en el marco de una preparación "integral", que implique los aspectos de los conocimientos científicos y prácticos que demanda el ejercicio de las profesiones en la sociedad, como los principios y valores éticos que conforman una sociedad justa y desarrollada.

- Promoverá la generación de condiciones de igualdad, sustentabilidad social, e implementación y fortalecimiento de la cultura de protección a los derechos humanos.

\section{Referencias Bibliográficas}

Asociación Nacional de Universidades e Instituciones de Educación Superior. (2000). La Educación Superior en el Siglo XXI Líneas estratégicas de desarrollo. www.anuies.mx/servicios/d estrategicos/...estrategicos/21/sXXI.pdf

Buquet Corleto, A., Cooper, J. A., Rodríguez Loredo, H., y Botello Lonngi, L. (2006). Presencia de Mujeres y Hombres en la UNAM: una radiografía. Recuperado de: http://www.pueg.unam.mx/images/pdf/radiografia.pdf

Centro de Estudios para el Adelanto de las Mujeres y la Equidad de Género. (2008). Análisis del Gasto Etiquetado para Mujeres y para Promover la Equidad de Género (Gemeg) en el Presupuesto de Egresos de la Federación (PEF). Recuperado de http://archivos.diputados.gob.mx/Centros_Estudio/ceameg/Inv_Finales_08/ DP2/2_4.pdf 
Comisión de Equidad de Género. (2007). Presupuesto Etiquetado para Mujeres y la Igualdad de Género 2008 (PEMIG 2008). Recuperado de http://www3.diputados.gob.mx/camara/content/download/149122/372567/fil e/3.pdf

Convención sobre la Eliminación de todas las Formas de discriminación contra la Mujer, Organización de las Naciones Unidas para la Igualdad de Género y el Empoderamiento de la Mujer § (1979).

Dawson, E. (2006). Handouts, Gender glossary. En Baker A. (Ed.), Gender Equality and Sexual Exploitation (pp.23)

García Máynez, E. (2005). Introducción al estudio del Derecho. México, D.F.: Editorial Porrua.

Gobierno de los Estados Unidos Mexicanos, Presidencia de la Republica. (2007). Plan Nacional de Desarrollo 2007-2012. Recuperado de http://pnd.calderon.presidencia.gob.mx/pdf/PND 2007-2012.pdf

Leach, F. (2003). Practising Gender Analysis in Education. Recuperado de http://policypractice.oxfam.org.uk/publications/download?ld=365288\&dl=http ://oxfamilibrary.openrepository.com/oxfam/bitstream/10546/115400/5/bkpractising-gender-analysis-education-010103-en.pdf

Ley General para la Igualdad entre Mujeres y Hombres, 2006, Cámara de Diputados del H. Congreso de la Unión § (2011).

Maceira Ochoa, L., Alva Mendoza, B. R., y Rayas Velasco L. (2007). Elementos para el análisis de los procesos de institucionalización de la perspectiva de género: una guía. México, D.F.: El Colegio de México.

Organización de las Naciones Unidas para la Educación la Ciencia y la Cultura. (2008). Priority Gender Equality Action Plan 2008-2011. Recuperado del Sitio de Internet United Nations Educational, Scientific and Cultural Organization: http://unesdoc.unesco.org/images/0018/001858/185856m.pdf

Organización de las Naciones Unidas. (2004). Transversalidad de género Informe de Desarrollo Humano México. Recuperado del sitio de Internet Programa de las Naciones Unidas para el Desarrollo México: 
http://www.undp.org.mx/desarrollohumano/eventos/images/Politica de gen ero.pdf

Overbeek, J. (1982). Population, an introduction. New York: Hardcourt Brace Jovanovich, INC.

Plan de Desarrollo Institucional 2009-2013, Universidad de Sonora § (2009)

Plan de Desarrollo Institucional 2009-2013, Universidad de Sonora § (2009)

Plan Estatal de Desarrollo 2009-2015, Poder Ejecutivo del Estado de Sonora § (2009)

Rees, T. (2002). Gender Mainstreaming: Misappropiated and Misunderstood. Suecia: Mimeo.

Rodríguez, R., Urquidi. L. y Pérez, A., (Coords.) (2010). La ciencia en Sonora, primeras aproximaciones. México: Universidad de Sonora.

Secretaria de Educación Pública (2007). Programa Sectorial de Educación 20072012. Recuperado de www.sep.gob.mx/work/appsite/prog sec.pdf 